\title{
Synergy of urokinase-type plasminogen activator receptor isomer (D1D2) and integrin $\alpha 5 \beta 1$ causes malignant transformation of hepatic cells and the occurrence of liver cancer
}

\author{
YING-QUN ZHOU ${ }^{1}$, XIAO-PING LV ${ }^{2}$, SHAN LI $^{1}$, BING BAI ${ }^{1}$ and LING-LING ZHAN ${ }^{1}$ \\ Departments of ${ }^{1}$ Clinical Laboratory and ${ }^{2}$ Gastroenterology, The First Affiliated Hospital of Guangxi Medical University, \\ Nanning, Guangxi 530021, P.R. China
}

Received December 13, 2013; Accepted June 17, 2014

DOI: $10.3892 / \mathrm{mmr} .2014 .2503$

\begin{abstract}
The aim of the present study was to investigate the correlations and possible synergy among the urokinase-type plasminogen activator receptor (UPAR) isomer D1D2 and integrin $\alpha 5 \beta 1$ expression levels, malignant transformation in hepatic cells and the occurrence of liver cancer. The expression site and concentration of UPAR (D1D2) were analyzed using polymerase chain reaction and in situ hybridization at the gene level in 60 samples of hepatocellular carcinoma (HCC) tissues, 60 samples of para-carcinoma tissues and 25 samples of normal liver tissues. The mRNA levels of uPAR (D1D2) and integrin $\alpha 5 \beta 1$ were markedly increased para-carcinoma tissue and liver cancer tissue as compared with those in normal tissue. The grey values of the three groups were significantly different $(\mathrm{P}<0.05)$. In situ hybridization revealed that the expression levels of uPAR (D1D2) and integrin $\alpha 5 \beta 1$ in the cytoplasm and the positive rate of the two molecules in the HCC tissue were significantly higher than those in the para-carcinoma and normal liver tissues, and the expression levels were positively correlated $\left(r_{s} 1=0.257, P<0.05 ; r_{s} 2=0.261, P<0.05\right)$. The results suggested that uPAR (D1D2) mRNA overexpression may be due to changes in the conformation of the UPAR isomer. Synergy of uPAR (D1D2) mRNA and integrin $\alpha 5 \beta 1$ interaction may result in abnormal signal transduction in liver cells and ultimately liver cell abnormal clonal hyperplasia and malignant transformation.
\end{abstract}

Correspondence to: Professor Ling-Ling Zhan, Department of Clinical Laboratory, The First Affiliated Hospital of Guangxi Medical University, 6 Shuangyong Road, Nanning, Guanxi 530021, P.R. China

E-mail: 13197614098@163.com

Key words: urokinase-type plasminogen activator receptor isomer D1D2, integrin $\alpha 5 \beta 1$, cell signal conduction, liver cell malignant transformation

\section{Introduction}

The extracellular matrix (ECM) is a complex three-dimensional network structure composed of macromolecules, including fibrous protein, proteoglycans and glycoproteins. The ECM mediates cellular adhesion and is involved in the intracellular signal transduction pathway, which, in turn, affects the occurrence, development and metastasis of malignancies (1). The adhesive cell-cell and cell-matrix attraction may be affected and modified when the cell undergoes malignant transformation.

A demonstrated that signal transduction of the urokinase plasminogen activator receptor (UPAR) activates the Ras-mitogen-activated protein kinase (MAPK) signaling pathway through the activity of a series of proteases (2). High expression levels of uPA and UPAR may result in the activation of integrin $\alpha 5 \beta 1$ and initiate a signaling cascade by aggregating the epidermal growth factor receptor. The signaling cascade subsequently causes a sustained and high level of extracellular signal-regulated kinase (ERK) activity and tumorigenicity (3). Integrin $\alpha 5 \beta 1$ is the most important transmembrane receptor in the uPAR signaling pathway, and may promote the development of malignant lesions, invasion and metastasis. UPAR, a heavily glycosylated single-chain glycoprotein, has three homologous domains: D1, D2 and D3. uPA dissociates these three regional proteins of uPAR into: uPAR (D1), comprised of the D1 domain, uPAR (D2D3), comprised of the D2 and D3 domains, uPAR (D1D2), comprised of the D1 and D2 domains, and the alternative splicing isomer of the uPAR gene, comprised of the D3 domain (4). In a previous study, the concentration and intensity of uPAR (D1D2) mRNA were found to be significantly increased in para-carcinoma and liver cancer tissue in primary culture as compared with normal tissue (5). The result indicated that uPAR (D1D2) mRNA overexpression may be due to UPAR isomer conformational changes, resulting in cell signal conduction abnormalities. In addition, the overexpression was closely associated with liver cell differentiation, abnormal clonal proliferation and increases in the degree of malignant transformation. Therefore, in the present study, the expression levels of uPAR (D1D2) and integrin $\alpha 5 \beta 1$ in normal, para-carcinoma and hepatic tissues 
Table I. PCR primers.

\begin{tabular}{lclcc}
\hline Gene & Primer type & \multicolumn{1}{c}{ Primer sequence } & No. of bases & Length (bp) \\
\hline uPAR (D1D2) & Forward & 5'-GACCTCTGCAGGACCACGAT-3' & 20 & 367 \\
& Reverse & 5'-GGTGGCGGTCATCCTTTG-3' & 18 & 337 \\
Integrin 05 & Forward & 5'-AAAAACGGGAAGCTCCAAGCCGCA-3' & 24 & 511 \\
& Reverse & 5'-AGGATGATGATCCACAGTGGGACG-3' & 24 & 24 \\
Integrin $\beta 1$ & Forward & 5'-GTGGAGAATGTATACAAGCAGGGC-3' & 24 \\
& Reverse & 5'-TTCCTGAGCTTAGCTGGTGTTGTG-3' & 24 & 21 \\
GAPDH & Forward & 5'-GGTGCTGAGTATGTCGTGGAG-3' & 22 & 292 \\
& Reverse & 5'-CAGTCTTCTGAGTGGCAGTGAT-3' & 22 & \\
\hline
\end{tabular}

PCR, polymerase chain reaction; bp, base pairs; uPAR, urokinase-type plasminogen activator receptor.

were detected by polymerase chain reaction (PCR) and in situ hybridization.

\section{Materials and methods}

Liver tissue sample collection. The collection of 60 hepatocellular carcinoma (HCC) tissue samples (from 42 males and 18 females) and 25 hemangioma tissue samples (from 18 males and 7 females), from patients with a pathological diagnosis and surgical resection, was conducted at the First Affiliated Hospital of Guangxi Medical University (Nanning, China) between December 2011 and April 2013. Prior to specimen collection, ethical informed consent was obtained from each patient. The study was approved by the Ethics Commitee of The First Affiliated Hospital of Guangxi Medical University (Nanning, China)

Liver cancer tissues were removed from the cancer tissues that were not yet necrotic, para-carcinoma tissues were extracted from areas surrounding the cancer tissues $\sim 2 \mathrm{~cm}$ and normal liver tissues were obtained from hepatic hemangioma patients undergoing surgical resection. One portion of each tissue sample was placed in a liquid nitrogen environment until mRNA extraction was performed, and another portion was fixed using neutral formalin solution, paraffin-embedded and sectioned.

Instruments and reagents. The following instruments and reagents were used: Ultraviolet Spectrophotometer (Nanodrap 2000; Thermo Fisher Scientific, Rockford, IL, USA), ultra-low temperature freezer (Thermo Forma 984; Thermo Fisher Scientific), Gel Doc ${ }^{\mathrm{TM}}$ XP + Gel imaging system, voltage steady flow electrophoresis apparatus (DYY-8B), thermal cycling machine (ABI Veriti ${ }^{\mathrm{TM}}$ ) and an inverted microscope (Nikon Corporation, Tokyo, Japan). Human uPAR (D1D2), integrin $\alpha 5$, integrin $\beta 1$, the in situ hybridization kit and 3\% hydrogen peroxide were purchased from Wuhan Boster Biological Techology, Ltd. (Wuhan, China). TRIzol, the RT-PCR kit and the nucleic acid dye were obtained from Treasure Biological Company (Dalian, China). The PCR kit and agarose were purchased from Thermo Fisher Scientific. Primers 1 and 4 were designed by Shanghai Sangon Biological Engineering Technology $\&$ Services Co., Ltd. (Shanghai, China) and synthesized by
Invitrogen Trading Co., Ltd. (Shanghai, China). Primers 2 (6) and 3 (7) were synthesized by Takara Bio, Inc. (Shiga, Japan).

Liver tissue RNA extraction. The liver tissues were removed from the liquid nitrogen. The required quantity of tissue was added to $1 \mathrm{ml}$ TRIzol and was then placed on ice for 5-10 min. The tissues were mechanically homogenized (JB50-S; Shanghai Heying Instrument Manufacturing Co., Ltd., Shanghai, China) and chloroform was added at a ratio of 5:1 lysate:chloroform (Shanghai Heying Instrument Manufacturing Co., Ltd.). The samples were left to rest for 5 min subsequent to being completely agitated and mixed, then centrifuged for $15 \mathrm{~min}(6,720 \mathrm{x} \mathrm{g})$ at $4^{\circ} \mathrm{C}$. DNA, protein and RNA were separated into sublayers, a white middle layer and an upper layer one after another.

The colorless upper layer of liquid was transferred to a new Eppendorf tube and an equal volume isopycnic isopropanol (Shanghai Heying Instrument Manufacturing Co., Ltd.) was added. The suspension was mixed, incubated at room temperature for $10 \mathrm{~min}$ and centrifuged for $15 \mathrm{~min}(5,600 \mathrm{x} \mathrm{g})$ at $4^{\circ} \mathrm{C}$.

The supernatant was discarded and the white sediment was retained. A volume of $1 \mathrm{ml} 75 \%$ absolute ethyl alcohol was added, the solution was pipetted gently and then centrifuged for $10 \mathrm{~min}(6,720 \mathrm{xg})$ at $4^{\circ} \mathrm{C}$. The alcohol was carefully discarded and the above procedure was repeated. The alcohol was added to an appropriate volume of diethylpyrocarbonate (DEPC; D100T; Sigma) to dissolve the RNA subsequent to drying in the air for $10 \mathrm{~min}$.

Measurement of RNA purity and PCR. The ultraviolet spectrophotometer was used to measure the concentration of RNA and the optical density (OD) value. The RNA purity was deemed to be at an adequate level if the OD260/OD280 value was between 1.8 and 2.0. The quantity of RNA was measured using the RT-PCR kit, according to the manufacturer's instructions. Reverse transcription was performed using an ABI Veriti Thermal Cycler instrument (Applied Biosystems, Foster City, CA, USA). The primers used are listed in Table I.

In situ hybridization. The in situ hybridization kit used a polyphase oligonucleotide probe corresponding to the target gene 
Table II. mRNA probe sequences of the target genes.

\begin{tabular}{ll}
\hline Target gene & mRNA probe sequence \\
\hline uPAR (D1D2) & 5'-TGTAA GACCAACGGG GATTG CCGTG TGGAAGAGTG-3' \\
5'-CACTC AGAGAAGACCAACAG GACCC TGAGC TATCG-3' \\
Integrin $\alpha 5$ & 5'-AGGAT GACCG CCACC TCCGT GGCTG TGGCT ACCTT-3' \\
& 5'-CAGTG CACCC CCATT GAATT TGACA GCAAA GGCTC-3' \\
Integrin $\beta 1$ & 5'-CAGGA GCAGA TTGCA GAATC TTATT ACCCC GAGTA-3' \\
& 5'-ACCAT CTTCC CCGCC ATGTT CAACC CAGAG GAGCG-3' \\
& 5'-AAATC TTGTG GAGAA TGTAT ACAAG CAGGG-3' \\
& 5'-CAGTC ACTGA AGAGT TCCAG CCTGT TTACA-3' \\
\hline
\end{tabular}

uPAR, urokinase-type plasminogen activator receptor.

labeled with digoxigenin. Table II shows the mRNA probe sequences of the target genes.

Cover slips and other vessels used in the in situ hybridization experiment were treated with water containing $1 \%$ DEPC to remove RNA enzymes prior to use. The experiment was conducted according the manufacturer's instructions of the kit and the results were observed under a microscope (Nikon, Tokyo, Japan).

Calculation of expression level scores. Positive expression of uPAR (D1D2) and integrin $\alpha 5 \beta 1$ was identified in the cytoplasm, exhibiting a yellow granular appearance. A total of 10 high power fields (HPFs) for each slice were randomly selected to determine the result and 100 tumor cells were counted to calculate the percentage of positive cells in each HPF. To comprehensively determine the expression levels of the two molecules, positive cell number and stained intensity scores were used. The positive cell score was calculated as follows: 0 points, number of positive cells $<10 \%$; 1 point, number of positive cells $<25 \%$; 2 points, number of positive cells $<50 \% ; 3$ points, number of positive cells $>50 \%$. The positive staining intensities were determined as follows: 0 points indicate negativity, consistent with the negative control; 1 point indicates low positive staining, appearing faint yellow (slightly more intense than the negative control); 2 points indicate positive staining, appearing medium yellow (more intense than the negative control); 3 points indicated marked positive staining, appearing tan or brown. The total score of each slide was calculated using the positive cell number score plus the stained intensity score: $0-2$ points signified low expression levels (-/+), 3-4 points signified medium expression levels $(++)$ and 5-6 points signified high expression levels $(+++)$.

Statistical analysis. The PCR results are expressed as gray values (mean \pm standard deviation). The in situ hybridization result was determined by positive cell number and staining intensity scores. SPSS version 17.0 (SPSS, Inc., Chicago, IL, USA) was used to process the data. Pairwise comparisons were calculated using one-way analysis of variance, categorical variable comparisons were performed with the $\chi^{2}$ test and correlation analysis was conducted using Spearman's rank correlation. $\mathrm{P}<0.05$ was considered to indicate a statistically significant difference.

\section{Results}

uPAR (D1D2) and integrin $\alpha 5 \beta 1 \mathrm{mRNA}$ expression levels. In the liver cancer and para-carcinoma HCC tissues and normal liver tissues, uPAR (D1D2; Fig. 1A), integrin $\alpha 5$ (Fig. 1C) and integrin $\beta 1$ (Fig. 1E) mRNA expression levels were determined using electrophoresis. The signal intensities in para-carcinoma and cancer tissues were markedly increased as compared with those in normal tissue. Water served as a blank control to exclude specific bands. uPAR (D1D2; Fig. 1B), integrin $\alpha 5$ (Fig. 1D) and integrin $\beta 1$ (Fig. 1F) samples served as internal reference bands corresponding to the three target genes. The function of these bands was to reduce the effect of differences in the doses of sample injection and the experimental results.

IMAGE J software analysis of each sample and the grey value of the corresponding internal reference. The expression levels of UPAR (D1D2) and integrin $\alpha 5 \beta 1$ mRNA, as determined by PCR, were calculated according to the following equation: Grey level ratio $=($ grey value of target band - grey value of water $) /($ grey value of internal reference - grey value of water) (Table III). mRNA levels of uPar (D1D2), integrin $\alpha 5$ and integrin $\beta 1$ were increased in para-carcinoma as compared with those in normal tissues, and even more increased in carcinoma tissues.

Detection of $u P A R(D 1 D 2)$, integrin $\alpha 5$ and integrin $\beta 1$ expression using in situ hybridization. In situ hybridization was observed under a microscope; positive signaling of the target genes was located in the cytoplasm and appeared as a tan color. The positive signal percentages for UPAR (D1D2), integrin $\alpha 5$ and integrin $\beta 1$ were highest in cancer tissues, followed by para-carcinoma tissues and were lowest in the normal liver tissues (Figs. 2-4). Subsequently, the expression levels of uPAR (D1D2), integrin $\alpha 5$ and integrin $\beta 1$ in the liver tissues were calculated from the microscopy results. The adopted positive cell and staining intensity scores were used to comprehensively determine the expression levels of the respective molecules (Table IV). 
Table III. Expression levels of uPAR (D1D2) and integrin $\alpha 5 \beta 1$.

\begin{tabular}{lllll}
\hline & & & \multicolumn{3}{c}{ Grey value ratio } \\
\cline { 3 - 5 } Group & $\mathrm{n}$ & uPAR (D1D2) & Integrin $\alpha 5$ & Integrin $\beta 1$ \\
\hline Normal tissue & 25 & $0.542 \pm 0.048$ & $0.509 \pm 0.070$ & $0.329 \pm 0.401$ \\
Adjacent tissue & 60 & $0.772 \pm 0.063^{\mathrm{b}}$ & $0.735 \pm 0.039^{\mathrm{b}}$ & $0.596 \pm 0.08^{\mathrm{b}}$ \\
Cancer tissue & 60 & $1.222 \pm 0.144^{\mathrm{a}}$ & $1.316 \pm 0.108^{\mathrm{a}}$ & $1.168 \pm 0.106^{\mathrm{a}}$ \\
\hline
\end{tabular}

${ }^{a} \mathrm{P}<0.05$ compared with normal tissue and para-carcinoma tissue. ${ }^{b} \mathrm{P}<0.05$ compared with para-carcinoma tissue and cancer tissue. uPAR, urokinase-type plasminogen activator receptor.
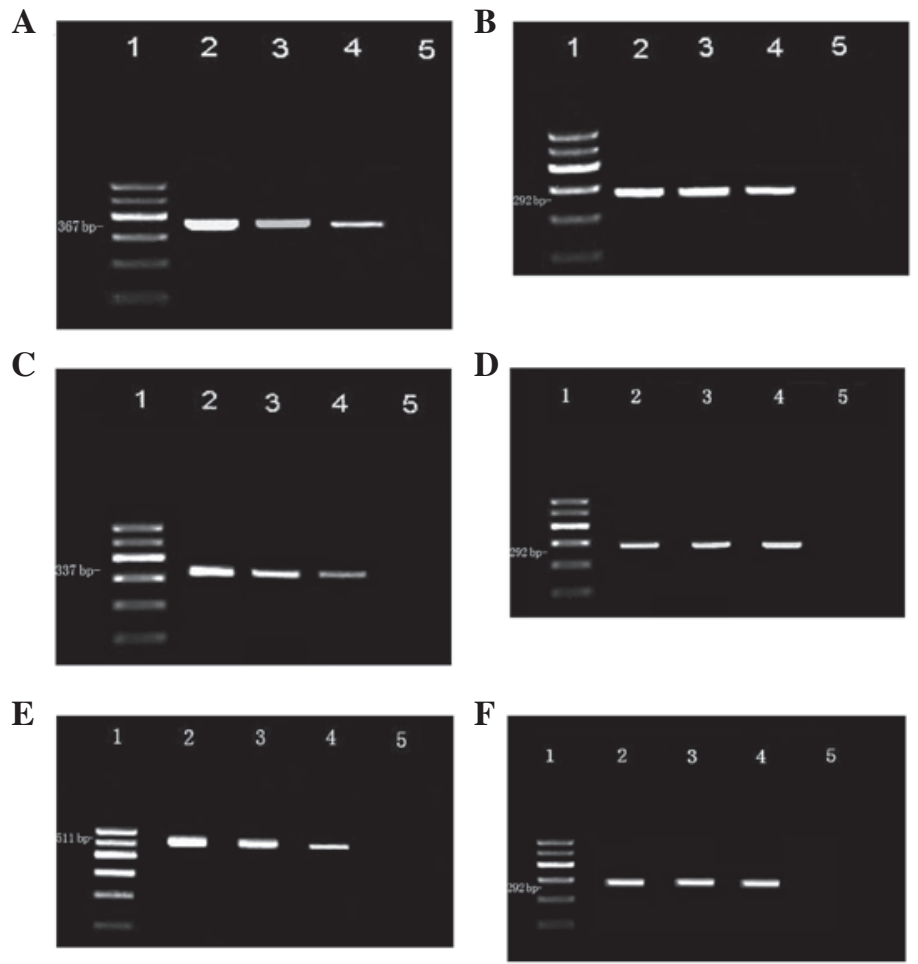

Figure 1. Electrophoresis results of uPAR isomer D1D2, integrin $\alpha 5$ and integrin $\beta 1$ detection in cancer, paracarcinoma and normal tissue. (A) uPAR (D1D2), (C) integrin $\alpha 5$ and (E) integrin $\beta 1$. Lane 1, marker; lane 2, cancer tissue; lane 3, para-carcinoma tissue; lane 4, normal tissue; lane 5, blank control. (B, D and F) indicate the internal reference bands corresponding to the three target genes: (B) uPAR (D1D2), (D) integrin $\alpha 5$ and (F) integrin $\beta 1$. uPAR, urokinase-type plasminogen activator receptor.

The correlations among the expression levels of uPAR (D1D2) and integrin $\alpha 5 \beta 1$ in HCC, and the clinical pathological features were determined to be as follows: The positive rate of UPAR (D1D2) in HCC was not correlated with $\alpha$ fetal protein (AFP), ferritin (SF), pathologic stage or tumor size, but was correlated with DNA copy number. When the DNA copy number was $>1.0 \times 10^{3}$, the positive percentage of uPAR (D1D2) was 90.7\%; When the DNA copy number was $<1.0 \times 10^{3}$, the positive percentage was $64.7 \%$ and a statistically significant difference was detected between the two groups $(\mathrm{P}<0.05)$. The integrin $\alpha 5 \beta 1$ expression levels did not correlate with AFP, SF, hepatitis B virus (HBV) DNA copy number or tumor size $(\mathrm{P}>0.05)$, but correlated with the pathologic stage. At different pathological stages, the percentages of positive integrin $\alpha 5 \beta 1$ in $\mathrm{HCC}$ were significantly different $(\mathrm{P}<0.05)$.
The correlation between the expression intensities of uPAR (D1D2) and integrin $\alpha 5 \beta 1$ in HCC tissues was positive. Thus, a synergistic effect between the expression levels of the two molecules may be considered $\left(\mathrm{r}_{\mathrm{s} 1}=0.257, \mathrm{P}<0.05 ; \mathrm{r}_{\mathrm{s} 2}=0.261\right.$, $\mathrm{P}<0.05$; Tables V and VI).

\section{Discussion}

Tumor transformation, growth, invasion and metastasis is a complex interaction process between tumor cells and the rest of the body. In this process, the various proteases adhered to the ECM (including serine proteases and matrix metalloproteinases) and growth factors (such as the integrin family and epidermal growth factor) are crucial (8).

Increasing evidence suggests that tumor-associated mRNA may induce tumor cells to produce special function proteins 


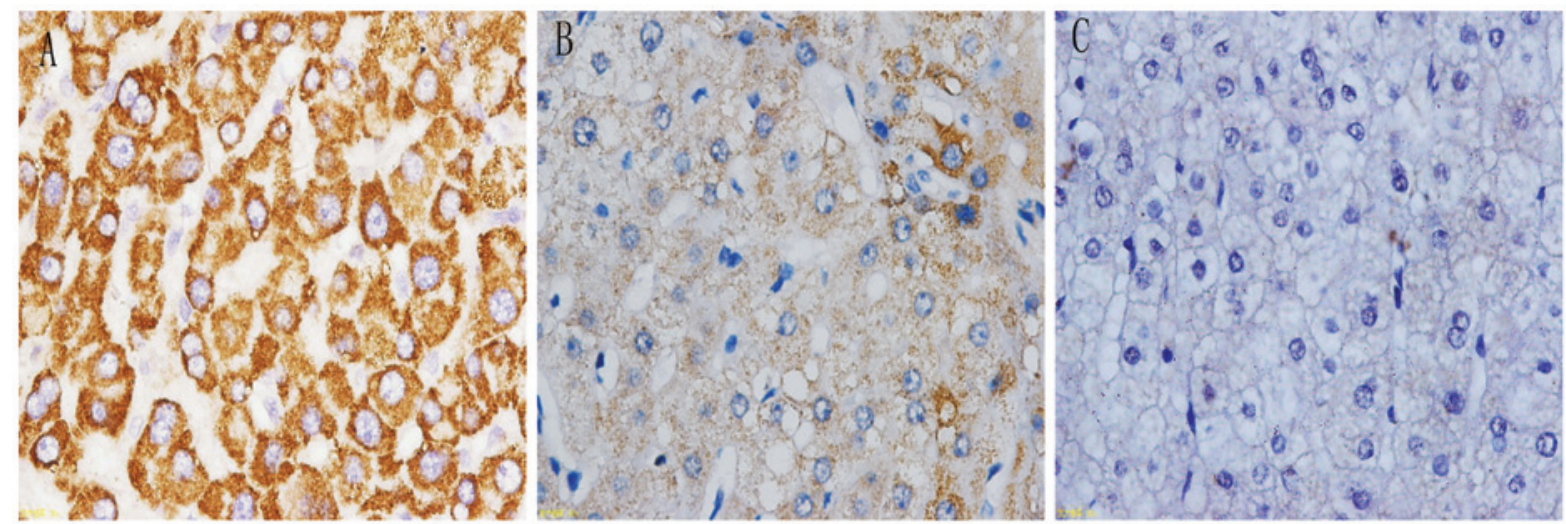

Figure 2. Expression of urokinase-type plasminogen activator receptor isomer D1D2 mRNA (tan color) in cancer, para-carcinoma and normal liver tissues (magnification, $\mathrm{x} 400$ ). (A) Cancer tissue; (B) paracarcinoma tissue; (C) normal tissue.

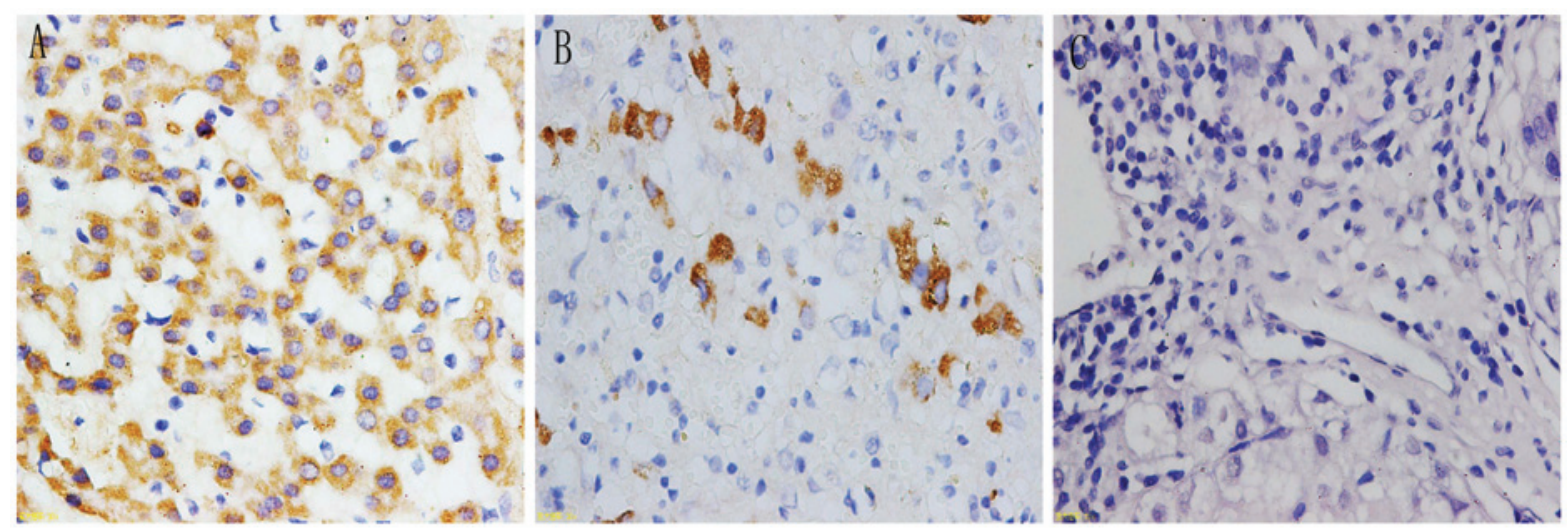

Figure 3. Expression of integrin $\alpha 5 \mathrm{mRNA}$ (tan color) in cancer tissue, adjacent tissue and normal tissue. (A-C) Integrin $\alpha 5 \mathrm{mRNA}$ expression in liver tissue (magnification, x400). (A) Cancer tissue; (B) adjacent tissue; (C) normal tissue.
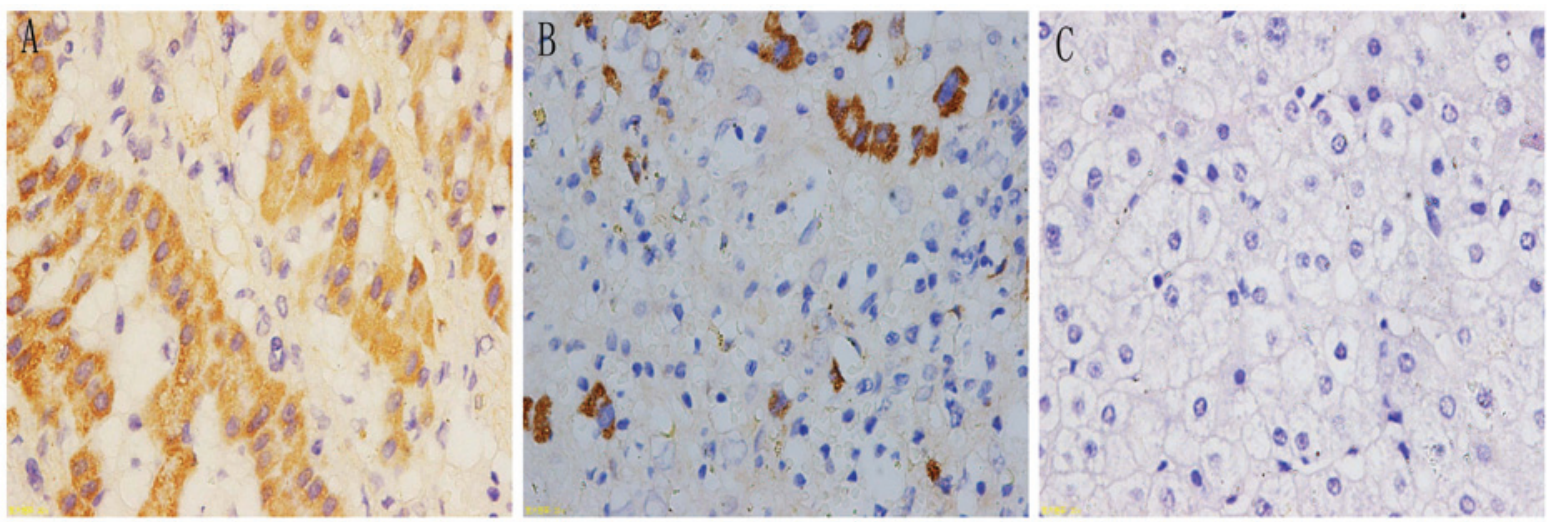

Figure 4. Expression of integrin $\beta 1 \mathrm{mRNA}$ (tan color) in cancer tissue, adjacent tissue and normal tissue. (A-C) Integrin $\beta 1 \mathrm{mRNA}$ expression in liver tissue (magnification, x400). (A) Cancer tissue; (B) adjacent tissue; (C) normal tissue.

closely associated with cancer occurrence and development. uPAR is an important factor in the urokinase system; certain studies have shown that UPAR mRNA is a specific marker of malignant transformation and metastasis in stomach cancer, colon cancer and non-small cell lung cancer (9-11). mRNA splice variants are considered specific sequences of diagnostic malignancy $(12,13)$, which may render certain protein areas missing, and subsequently result in loss or change of function, or the development of a novel function. There are multiple mRNA splice variants of uPAR; studies have demonstrated that these splice variants exert a marked effect in breast cancer, small cell lung cancer and other malignancies (14-17). In the present study, PCR and in situ hybridization techniques were used to detect the expression levels of uPAR (D1D2) mRNA in the liver cancer, para-neoplastic and normal tissues. The expression levels of the uPAR (D1D2) splice variant were observed to be significantly different in HCC, para-carcinoma and normal liver cells and in primary culture; the expression levels of uPAR (D1D2) exhibited 
Table IV. Expression levels of uPAR (D1D2), integrin $\alpha 5$ and integrin $\beta 1$ in liver tissue.

\begin{tabular}{|c|c|c|c|c|c|c|c|}
\hline \multirow[b]{2}{*}{ Gene } & \multirow[b]{2}{*}{ Group } & \multirow[b]{2}{*}{$\mathrm{n}$} & \multicolumn{4}{|c|}{ Staining results } & \multirow[b]{2}{*}{ Positive rate $(\%)$} \\
\hline & & & - & + & ++ & +++ & \\
\hline \multirow[t]{3}{*}{ uPAR (D1D2) } & Normal tissue & 25 & 16 & 6 & 3 & 0 & $36.0^{\mathrm{a}}$ \\
\hline & Adjacent tissue & 60 & 24 & 12 & 16 & 8 & $60.0^{\mathrm{b}}$ \\
\hline & Cancer tissue & 60 & 10 & 4 & 25 & 21 & 83.3 \\
\hline \multirow[t]{3}{*}{ Integrin $\alpha 5$} & Normal tissue & 25 & 22 & 3 & 0 & 0 & $12.0^{\mathrm{a}}$ \\
\hline & Adjacent tissue & 60 & 38 & 6 & 12 & 4 & $36.7^{\mathrm{b}}$ \\
\hline & Cancer tissue & 60 & 21 & 7 & 18 & 14 & 65.0 \\
\hline \multirow[t]{3}{*}{ Integrin $\beta 1$} & Normal tissue & 25 & 22 & 2 & 1 & 0 & $12.0^{\mathrm{b}}$ \\
\hline & Adjacent tissue & 60 & 37 & 8 & 10 & 5 & $38.3^{\mathrm{b}}$ \\
\hline & Cancer tissue & 60 & 23 & 6 & 15 & 16 & 61.7 \\
\hline
\end{tabular}

${ }^{\mathrm{a}} \mathrm{P}<0.05$ compared with normal and para-carcinoma tissues. ${ }^{\mathrm{b}} \mathrm{P}<0.05$ compared with para-carcinoma and cancer tissues. uPAR, urokinase-type plasminogen activator receptor.

Table V. Correlation between uPAR (D1D2) expression and integrin $\alpha 5$ expression in HCC.

\begin{tabular}{|c|c|c|c|c|c|}
\hline \multirow{2}{*}{$\begin{array}{l}\text { Integrin } \alpha 5 \\
\text { expression }\end{array}$} & \multicolumn{4}{|c|}{ uPAR (D1D2) expression } & \multirow[b]{2}{*}{ Total } \\
\hline & - & + & ++ & +++ & \\
\hline- & 7 & 1 & 8 & 5 & 21 \\
\hline+ & 1 & 0 & 4 & 2 & 7 \\
\hline++ & 0 & 2 & 9 & 7 & 18 \\
\hline+++ & 2 & 1 & 4 & 7 & 14 \\
\hline Total & 10 & 4 & 25 & 21 & 60 \\
\hline
\end{tabular}

uPAR, urokinase-type plasminogen activator receptor; HCC, hepatocellular carcinoma.

Table VI. Correlation between uPAR (D1D2) expression and integrin $\beta 1$ expression in HCC.

\begin{tabular}{|c|c|c|c|c|c|}
\hline \multirow{2}{*}{$\begin{array}{l}\text { Integrin } \beta 1 \\
\text { expression }\end{array}$} & \multicolumn{4}{|c|}{ uPAR (D1D2) expression } & \multirow[b]{2}{*}{ Total } \\
\hline & - & + & ++ & +++ & \\
\hline- & 5 & 1 & 13 & 4 & 23 \\
\hline+ & 2 & 1 & 1 & 2 & 6 \\
\hline++ & 1 & 1 & 6 & 7 & 15 \\
\hline+++ & 2 & 1 & 5 & 8 & 16 \\
\hline Total & 10 & 4 & 25 & 21 & 60 \\
\hline
\end{tabular}

uPAR, urokinase-type plasminogen activator receptor; HCC, hepatocellular carcinoma.

an upward trend with liver cancer development. The expression intensity of uPAR (D1D2) in HCC was not associated with AFP, $\mathrm{SF}$, pathological stage or tumor size, but was associated with the HBV DNA copy number $(\mathrm{P}<0.05)$. This is consistent with the previous results of primary cultured hepatoma cells. Continuous replication $\mathrm{HBV}$ activates particular proto-oncogenes, but renders certain tumor-suppressor genes inactive and mutational, thus promoting the occurrence of cancer. The integration of virus DNA may increase the occurrence of the HBV $\mathrm{X}$ antigen and induce malignant transformation of liver cells (18).

Integrins are a group of transmembrane glycoprotein receptors widely distributed on cell surfaces. In addition to 
mediating adhesion between the cell and the ECM, integrins affect ECM degradation and tumor cell chemotaxis, proliferation, apoptosis and metastasis (19). Integrin $\alpha 5 \beta 1$ is an important member of the integrin family. A number of studies have demonstrated the association between integrin $\alpha 5 \beta 1$ and numerous types of cancer, including liver, breast and colorectal cancer (20-21). Furthermore, uPA and uPAR, overexpressed in cancer cells, frequently combine with integrin $\alpha 5 \beta 1$ to form uPA-uPAR- $\alpha 5 \beta 1$ complexes, which induce the activation of signaling pathways to alter cell adhesion, proliferation and migration (22). Aguirre Aguirre Ghiso et al (23) considered the interaction between uPAR and integrin $\alpha 5 \beta 1$ on the cell surface to induce a series of reactions and this interaction may be involved in MAPK-ERK signaling pathway activation, eventually resulting in head and neck tumors, as determined in head and neck cancer models. Wei et al (24) deemed that the binding between uPAR and $\alpha 5 \beta 1$ requires maximum fibrin and tumor cell invasion, for which enhancement of the $\mathrm{Src} / \mathrm{Rac} /$ ERK signaling pathway is necessary. In the present study, PCR and in situ hybridization were employed to detect the expression levels of integrin $\alpha 5$ and integrin $\beta 1$ mRNA in HCC, para-carcinoma and normal liver tissues. Similar findings to those for uPAR (D1D2) were obtained; integrin $\alpha 5 \beta 1$ expression levels in HCC tissues were higher than those in para-carcinoma and normal liver tissues. Thus, the expression levels of integrin $\alpha 5 \beta 1$ in $\mathrm{HCC}$ were associated with the pathological stage, and the associations among integrin $\alpha 5 \beta 1$ expression levels, liver cancer differentiation and malignant transformation are inextricable. The results also revealed that uPAR (D1D2) and integrin $\alpha 5 \beta 1$ expression levels in HCC were positively correlated, and exerted a synergistic effect in cancer occurrence and development. The correlation between uPAR (D1D2) expression levels and liver cancer malignant transformation was initially observed in a previous study (5), but the correlation between integrin $\alpha 5 \beta 1$ expression levels and liver cancer, and the synergistic effect of uPAR (D1D2) and integrin $\alpha 5 \beta 1$ in $\mathrm{HCC}$ had not yet been reported, to the best of our knowledge.

In conclusion, the results of the present study demonstrated that the synergy of uPAR (D1D2) and integrin $\alpha 5 \beta 1$ expression in hepatocytes was closely associated with the occurrence of liver cancer and subsequent metastasis. The cell signal transduction pathways and gene therapy as anticancer strategies have become a predominant focus of cancer research. It has been found that the cell signal transduction pathways of different types of uPAR isomers and integrin- $\alpha 5 \beta 1$ are correlated with tumorigenesis but the mechanism has not been fully elucidated. Thus further studies will aim to determine the underlying mechanisms.

\section{References}

1. Stetler-Stevenson WG, Aznavoorian S and Liotta LA: Tumor cell interactions with the extracellular matrix during invasion and metastasis. Annu Rev Cell Biol 9: 541-573, 1993.

2. Scaltriti $\mathrm{M}$ and Baselga J: The epidermal growth factor receptor pathway: a model for targeted therapy. Clin Cancer Res 12: 5268-5272, 2006.

3. Liu D, Aguirre Ghiso J, Estrada Y and Ossowski L: EGFR is a transducer of the urokinase receptor initiated signal that is required for in vivo growth of a human carcinoma. Cancer Cell 1: 445-457, 2002.
4. Piironen T, Laursen B, Pass J, et al: Specific immunoassays for detection of intact and cleaved forms of the urokinase receptor. Clin Chem 50: 2059-2068, 2004.

5. Zhou Y, Lu X, Li S and Zhan L: Correlation between the overexpression of urokinase receptor isoform uPAR (D1D2) and hepatic cell malignant transformation. Mol Med Rep 9: 1689-1696, 2014.

6. Hsu SL, Chenga CC, Shi YR and Chiang CW: Proteolysis of integrin $\alpha 5$ and $\beta 1$ subunits involved in retinoic acid-induced apoptosis in human hepatoma Hep3B cells. Cancer Lett 167: 193-204, 2001.

7. Barillari G, Albonici L, Incerpi S, et al: Inflammatory cytokines stimulate vascular smooth muscle cells locomotion and growth by enhancing $\alpha 5 \beta 1$ integrin expression and function. Atherosclerosis 154: 377-385, 2001.

8. Bezdenezhnykh N, Semesiuk N, Lykhova O, et al: Impact of stromal cell components of tumor microenvironment on epithelial-mesenchymal transition in breast cancer cells. Exp Oncol 36: 72-78, 2014

9. Kita Y, Fukagawa T, Mimori K, et al: Expression of uPAR mRNA in peripheral blood is a favourite marker for metastasis in gastric cancer cases. Br J Cancer 100: 153-159, 2009.

10. Dang J, Wang Y, and Doe WF: Sodium butyrate inhibits expression of urokinase and its receptor mRNAs at both transcription and post-transcription levels in colon cancer cells. FEBS Lett 359: 147-150, 1995.

11. Montuori N, Mattiello N, Mancini A, et al: Urokinase-mediated posttranscriptional regulation of urokinase-receptor expression in non small cell lung carcinoma. Int J Cancer 105: 353-360, 2003.

12. Groenendijk FH, Zwart W, Floore A, Akbari S and Bernards R: Estrogen receptor splice variants as a potential source of false-positive estrogen receptor status in breast cancer diagnostics. Breast Cancer Res Treat 140: 475-484, 2013.

13. Tian T, Shu Y, Chen J, et al: A functional genetic variant in microRNA-196a 2 is associated with increased susceptibility of lung cancer in Chinese. Cancer Epidem Biomarkers Prev 18: 1183-1187, 2009.

14. Grismayer B, Sato S, Kopitz C, et al: Overexpression of the urokinase receptor splice variant uPAR-del $4 / 5$ in breast cancer cells affects cell adhesion and invasion in a dose-dependent manner and modulates transcription of tumor-associated genes. Biol Chem 393: 1449-1455, 2012.

15. Sato S, Kopitz C, Grismayer B, et al: Overexpression of the urokinase receptor mRNA splice variant uPAR-del $4 / 5$ affects tumor-associated processes of breast cancer cells in vitro and in vivo. Breast Cancer Res Treat 127: 649-657, 2011.

16. Almasi CE, Drivsholm L, Pappot H, Høyer-Hansen G and Christensen IJ: The liberated domain I of urokinase plasminogen activator receptor - a new tumour marker in small cell lung cancer. APMIS 121: 189-196, 2013.

17. Parise LV, Lee $J$ and Juliano RL: New aspects of integrin signaling in cancer. Semin Cancer Biol 10: 407-414, 2000.

18. Yao M, Zhou XD, Zha XL, et al: Expression of the integrin $\alpha 5$ subunit and its mediated cell adhesion in hepatocellular carcinoma. J Cancer Res Clin Oncol 123: 435-440, 1997.

19. Korah R, Boots M, and Wieder R: Integrin $\alpha 5 \beta 1$ promotes survival of growth-arrested breast cancer cells: an in vitro paradigm for breast cancer dormancy in bone marrow. Cancer Res 64: 4514-4522, 2004.

20. Jayne DG, Heath RM, Dewhurst O, Scott N and Guillou PJ: Extracellular matrix proteins and chemoradiotherapy: $\alpha 5 \beta 1$ integrin as a predictive marker in rectal cancer. Eur J Surg Oncol 28: 30-36, 2002.

21. Su Q, Schröder CH, Hofmann WJ, et al: Expression of hepatitis B virus X protein in HBV-infected human livers and hepatocellular carcinomas. Hepatology 27: 1109-1120, 1998.

22. Ossowski L and Aguirre-Ghiso JA: Urokinase receptor and integrin partnership: coordination of signaling for cell adhesion, migration and growth. Curr Opin Cell Biol 12: 613-620, 2000.

23. Aguirre Ghiso JA, Kovalski K and Ossowski L: Tumor dormancy induced by downregulation of urokinase receptor in human carcinoma involves integrin and MAPK signaling. J Cell Biol 147: 89-104, 1999.

24. Wei Y, Tang CH, Kim Y, et al: Urokinase receptors are required for $\alpha 5 \beta 1$ integrin-mediated signaling in tumor cells. J Biol Chem 282: 3929-3939, 2007. 